\title{
Personalized Distance Learning Using the STACK System
}

\author{
Dmitry V. Kruchinin, Yuriy V. Shablya, Alexander A. \\ Shelupanov \\ Department of Complex Information Security of Computer \\ Systems \\ TUSUR University \\ Tomsk, Russia \\ kdv@keva.tusur.ru, syv@keva.tusur.ru, saa@keva.tusur.ru
}

\author{
Vadim S. Melman \\ Department of Information Systems Security \\ TUSUR University \\ Tomsk, Russia \\ vadiamylman@yandex.ru
}

\begin{abstract}
In this paper, we consider aspects of organizing self-learning for students on mathematical disciplines. We describe a scheme for monitoring and evaluating work of students based on using a specific feedback. That allows us to personalize distance learning. We demonstrate the advantages of learning systems like the STACK system that is based on computer algebra systems.
\end{abstract}

Keywords—distance learning; self-learning; STACK; feedback; computer algebra system

\section{INTRODUCTION}

Learning activity as the process of obtaining new knowledge and skills is an essential part of the life of any person. Every day, when we are faced with something unknown, we have to start its researching and studying. For effective organization of the learning activity, in most countries there are education systems, each of which has its own characteristics.

In the modern world, the global informatization of society is gradually making its changes, which also affect the educational process. Today, more and more attention is paid to distance e-learning. For example, there is a whole set of platforms for organizing e-learning courses, such as Moodle [1], Docebo [2], Efront [3], etc. At the same time, each platform has its own advantages and disadvantages in comparison with the rest.

Distance e-learning has already shown its effectiveness in the organization of self-learning, when the student has sufficient motivation for studying the discipline and is able to absorb new knowledge fast and qualitatively. Also, distance elearning can be effectively used for organizing students' individual work.

However, if we consider the process of solving mathematical tasks, we find the problem of interpreting answers introduced by students. Most distance e-learning platforms offer only a simple comparison of the input answer with the answer computed in the system. In this case, the answer is considered as the correct answer only when they completely coincide. This problem limits the possibilities of studying mathematical disciplines by using the technology of distance e-learning.

We can distinguish the following shortcomings of modern e-learning systems relative to the studying mathematical disciplines:

- Some mathematical tasks can have a solution in the form of a whole set of feasible solutions. Thus, there is a high probability that a student will enter the answer that does not coincide with the answer computed in the system, but both answers are equivalent to each other. In this case, the system will give an erroneous conclusion that the solution is not true.

- If there is an error in the calculations, the final answer will be incorrect. Hence, the system will give a conclusion that the solution is completely wrong.

- If we generate one or more variants for each typical mathematical task, eventually the tasks' answers will be shared among students. It is caused by the huge number of students enrolled in distance e-learning courses and their active use of Internet resources. Therefore, in order to improve the quality of education, it is necessary to regularly update the tasks. In this case, an ideal situation is when there is an individual variant of tasks for each student, i.e. each task is personalized. But then it is necessary to spend much more resources on generating tasks and obtaining tasks' solutions for their subsequent estimation.

- Most e-learning systems provide conclusions about the correctness of the answer or the value of the correct answer for the task. Sometimes in the e-learning systems we can find theoretical information about the task, the detailed solution of the task or of a typical task, i.e. either there is a complete detailed solution, or it is missing. But it is more effective when a student himself understands the solution of a task with some tips (providing a specific feedback).

Thus, considering above mentioned shortcomings of modern e-learning systems relative to the studying mathematical disciplines, the purpose of this work is to develop an approach for organizing self-learning for students which allows us to personalize the generation, checking and estimation of students' work with specific feedbacks. 
As the basis for the approach, we propose to use the following scheme for organizing students' work (Fig. 1):

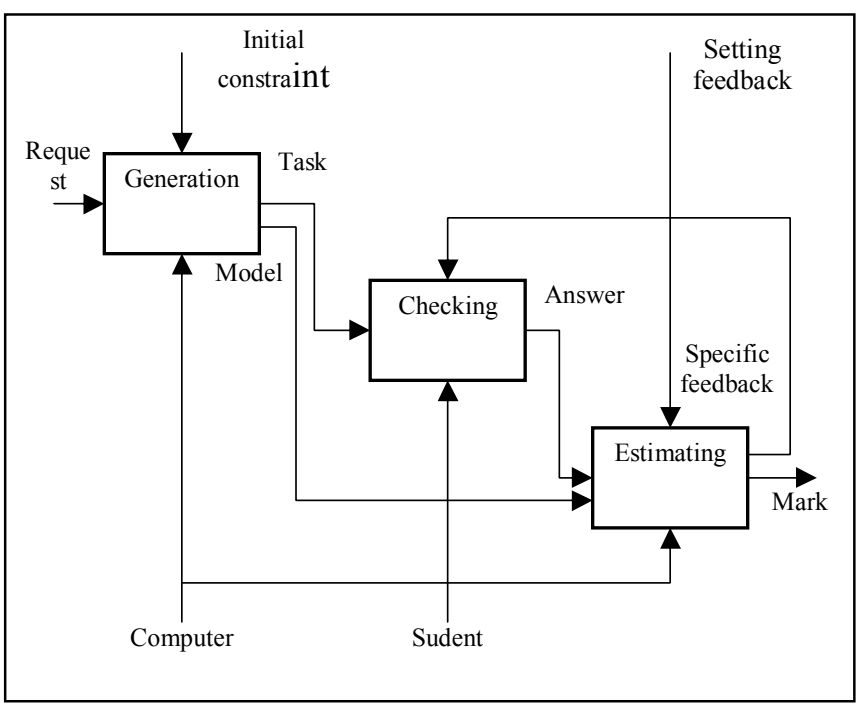

Fig. 1. Scheme for organizing students' work.

The approach consists of the organization of several stages: Generation, checking, and estimation that describe bellow. The main emphasis is to generate a feedback generation and personalize it. For that, firstly, we need to generate personal tasks. Secondly, we need to realize the analysis of the student's answer.

\section{Personalized Generating AND CHECKING A TASK}

For personalized generating and checking the solution of mathematical tasks, we propose to use a set of the following software:

- Maxima computer algebra system [4], which has great functionality for solving and programming mathematical tasks of any complexity.

- TeX typesetting system with LaTeX macro package [5], which has great functionality for making and printing texts of any complexity.

The choice of such a set of software is determined by the following advantages:

- Maxima and LaTeX are distributed freely and do not require the purchase of licenses for their use.

- Maxima and LaTeX are well-checked by time software, as their development has been under way for decades, and they are constantly being updated and updated;

- LaTeX is the standard for the design of most foreign scientific journals in the mathematical and technical sciences. LaTeX does great with displaying mathematical formulas;

- Maxima is able to implement most of all functions of mathematics and programming, which allows us to generate almost any mathematical task;

- Maxima is able to translate a result of calculations into the TeX format;
- The style file maxiplot.sty [4] is able to embed the Maxima code in LaTeX documents. Then there is a possibility of combined use of Maxima and LaTeX. That is, using LaTeX we formalize a process of solving mathematical tasks, and within the framework of this solving we use Maxima for calculations.

Thus, Maxima and LaTeX are perfectly interact with each other, and on their basis it is possible to realize the automation of personalized generating mathematical tasks and checking solutions of the generated tasks.

\section{Personalized FEedBACK AND ESTIMATING A TASK}

In addition to generating and checking mathematical tasks, we need to organize a system for estimating a student's solution of the task and to provide personalized feedback. Taking into account the above suggestions about using Maxima and LaTeX for generating and checking the solution of mathematical tasks, we propose to use the System for Teaching and Assessment using a Computer algebra Kernel (STACK) [6] that is an open-source system for computer-aided assessment in Mathematics performed on the Moodle platform.

STACK concentrates on student-provided answers which are mathematical expressions. For example, a student might respond to a question with a polynomial or matrix. Essentially STACK asks for mathematical expressions and evaluates these using computer algebra. The prototype test is the following pseudo-code [7]

If

simplify(student_answer-teacher_answer) $=0$

then

$\operatorname{mark}=1$,

else

$\operatorname{mark}=0$.

STACK uses a computer algebra system Maxima and TeX to implement generating tasks and checking students' answers. The computer algebra system provides a group of functions that allow us manipulate students' answers and generate outcomes such as providing specific feedback.

The idea of the specific feedback is to localize the mistake of solving by generating a potential response tree (Fig.2, Fig.3), which is responsible for the scoring. For one task, it is possible to specify several inputs (student answers to the task) and several potential response trees, respectively. The potential response tree consists of nodes in which the value of the student's input is compared with the model specified during generating the task. Depend on the node STACK displays a different feedback. The answer and the model could be compared by number of functions, for example by algebraic equivalence. Using this property, it is possible to give a personalized feedback and estimation. 


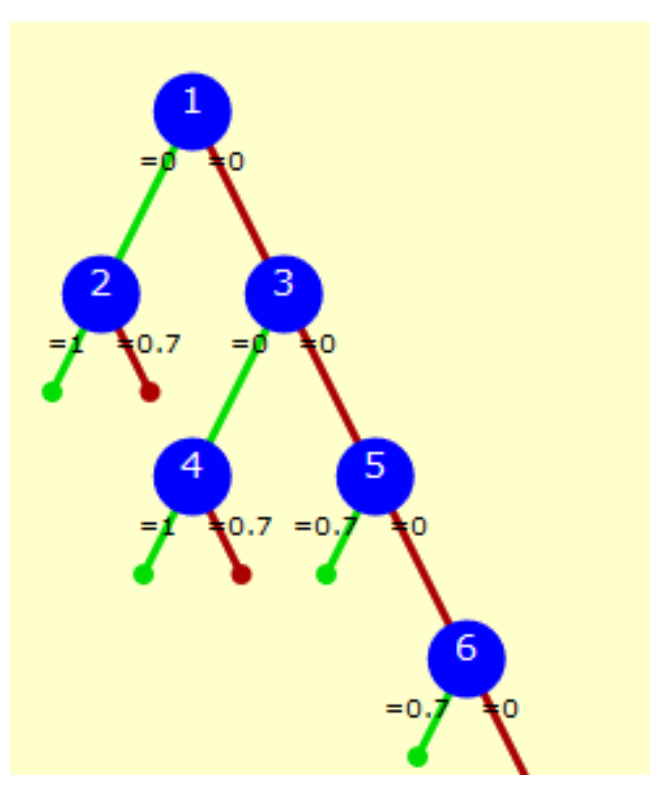

Fig.2 Scheme for potential response tree.

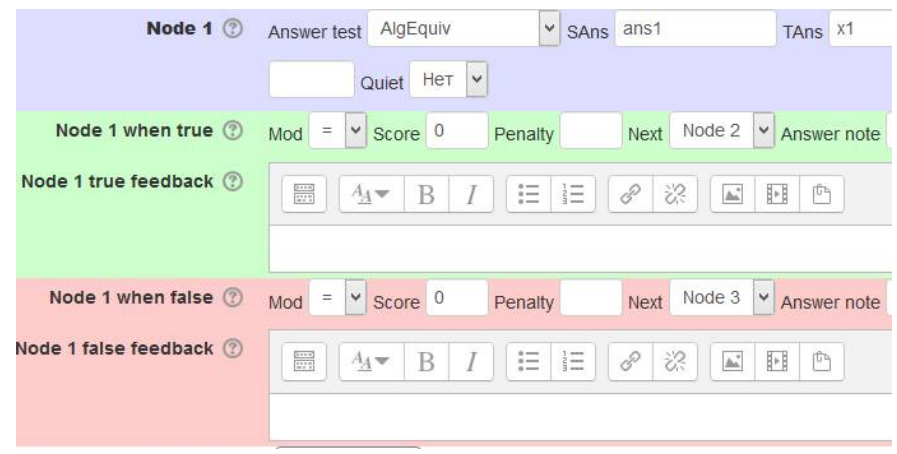

Fig.3 Options for potential response tree.

Each potential response tree computes a score from 0 to 1 , which is then multiplied by the value of question-input for the response tree. If the resulting score (let's call it $s$ ) lies outside this range, then it is assumed to be $\min (\max (s, 0), 1)$.

Since the student has several attempts to enter an aswer when solving a task, it is possible to set a penalty for incorrect attempts. Since there is a potential response tree, it is possible to organize personalized penalty by setting a value of the penalty in each node of the potential response tree.

Denote the sum of the score after traversing a potential response tree for the current attempt by $s k$, and denote the penalty by $p k$. Then for each attempt $k$ there hold: - if $s k=1$ then $p k=0$;

- if the penalty $p$ in the last node is not NULL then $p k=p$;

- else the default value (usually it is $10 \%=0.1$ ).

The final score for the task is the sum of the values of all potential response trees minus penalty of all attempts.

\section{RESUlts}

Using the proposed approach, we've developed about 500 generated templates for mathematical tasks. It allow us to construct e-course for the subject Calculus 1 for the 1-grade students. The course is construct on the Moodle platform. It is included 50 quizzes on Limits, Differentiation, and some basic knowledges. There are 2 types of quizzes: with general feedbacks and without. By general feedback, we mean worked solution for each task. That allow us to construct the whole system of education. Preliminary results show an improvement in the quality of students' assimilation of educational material.

\section{CONCLUSION}

Using e-courses developed on the proposed approach, it becomes possible to personalize the assessments of students. , That is, it's possible to give the most correct and fair assessment, depending on the degree of correctness of the answer.

Due to individual students' work with each task, the quality of evaluation approaches the teacher's personal assessment, that has a positive effect on the student's progress and motivation.

\section{ACKNOWLEDGMENT}

This work was partially supported by the Ministry of Education and Science of Russia, Government Order no. 2.8172.2017/BP (TUSUR).

\section{REFERENCES}

[1] Moodle. https://moodle.org/

[2] Docebo. https://www.docebo.com/

[3] Efront. https://www.efrontlearning.com/

[4] Maxima, a Computer Algebra System. http://maxima.sourceforge.net/

[5] Latex. https://www.latex-project.org//

[6] Stack. https://moodle.org/plugins/qtype_stack

[7] Chris Sangwin. Computer Aided Assessment of Mathematics, , Oxford University Press, 2013. 